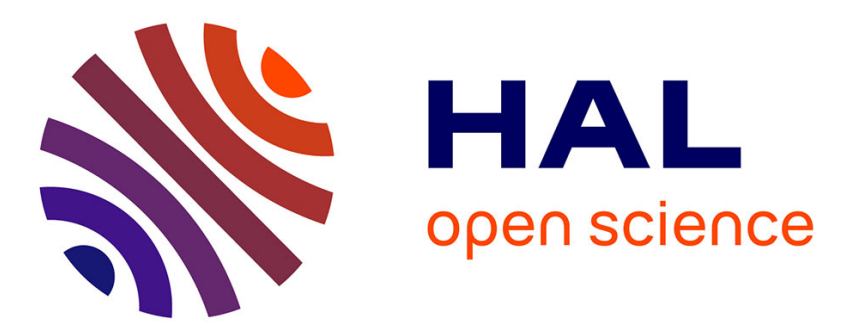

\title{
Lessons from Tarceva in pancreatic cancer: where are we now, and how should future trials be designed in pancreatic cancer?
}

\author{
Pierre Laurent-Puig, Julien Taieb
}

\section{- To cite this version:}

Pierre Laurent-Puig, Julien Taieb. Lessons from Tarceva in pancreatic cancer: where are we now, and how should future trials be designed in pancreatic cancer?. Current Opinion in Oncology, 2008, 20 (4), pp.454-8. 10.1097/CCO.0b013e32830218d6 . hal-00309684

\section{HAL Id: hal-00309684 https://hal.science/hal-00309684}

Submitted on 7 Aug 2008

HAL is a multi-disciplinary open access archive for the deposit and dissemination of scientific research documents, whether they are published or not. The documents may come from teaching and research institutions in France or abroad, or from public or private research centers.
L'archive ouverte pluridisciplinaire HAL, est destinée au dépôt et à la diffusion de documents scientifiques de niveau recherche, publiés ou non, émanant des établissements d'enseignement et de recherche français ou étrangers, des laboratoires publics ou privés. 
Lessons from Tarceva in pancreatic cancer: where are we now, and how should future trials be designed in pancreatic cancer?

Pierre Laurent-Puig ${ }^{1,2,3,4}$ Julien Taieb $^{1,3}$

${ }^{1}$ Université Paris Descartes, Paris France

${ }^{2}$ Inserm UMR-S 775 Molecular basis of response to xenobiotics

${ }^{3}$ Assistance Publique - hopitaux de Paris, Hopital Européen Georges Pompidou, 75015 Paris

${ }^{4}$ Corresponding Author: Pierre Laurent-Puig U775 INSERM, 45 rue des SaintsPères 75006 Paris

pierre.laurent-puig@univ-paris5.fr

Purpose of review: The recent advances in the use of targeted therapy in pancreatic cancer are based on the knowledge of genetic alterations that occur during pancreatic carcinogenesis. We describe the repository of frequent alterations targeting tumor suppressor genes and oncogenes. We focus our attention on the EGFR signaling pathway, which can be activated through different alterations and seems to play a central role in the cell transformation. Multiple targeted drugs have been developed against different partners of this network trying to improve the treatment of pancreatic cancer patients. Recent findings: Tarceva has obtained approval in the USA and Europe for metastatic pancreatic cancer with a modest increase of median survival and a $6 \%$ increase in one-year survival rates, suggesting that only a small fraction of patients truly benefit of it. The comparison with lung and colon cancer suggests that KRAS mutations could be a predictive marker of resistance. Other promising drugs targeting different partner of the EGFR signalling pathway could play a synergistic role with tarceva as inhibitors of mTOR, MEK, and NFKB or can directly turn down RAS. Summary: The biology of EGFR, MAPK, $\mathrm{PI} 3 \mathrm{~K} / \mathrm{mTOR}$ network suggests that combination of drugs targeting simultaneously different partners should improve survival.

Keywords : Pancreatic cancer, genetic alterations, targeted therapy EGFR signaling pathway, Mutation, KRAS, mTOR, NFkB, PIK3CA 


\section{Introduction}

As cancer is caused by alteration in oncogenes, tumor suppressor genes and microRNA ${ }^{[1]}$, the knowledge of the these alterations should be of help to understand the mechanisms of cell transformation and also to find appropriate targeted anticancer treatments. Among human solid tumors, pancreatic cancer is one of the most studied for the genetic alterations that occurs during cell transformations. Incidence and mortality rate (between 5 and 11 to 100000 inhabitants) are close underlying the extreme severity of this disease. Until a recent time, the only treatment with an impact on survival was surgery. In the palliative setting gemcitabine remains since 1997 the standard treatment despite poor response rate and survivals. The recent introduction of targeted therapies in the therapeutic armamentarium against cancer raised some hopes in the treatment of patients with advanced or metastatic diseases. The knowledge of the molecular events occurring in the malignant transformation processes should allow the development of efficient targeted therapies.

\section{Chromosomal instability in pancreatic cancer}

This tumour is not only homogeneous in its clinical presentation, but also in its histological characteristics. Although biliary epithelium represents less than $5 \%$ of the whole pancreatic cells, more than $90 \%$ of pancreatic cancers are adenocarcinoma coming form the malignant transformation of this epithelium. The particular aggressiveness of these tumours is owing to the existence of an important genetic plasticity of the pancreatic tumour cells. Indeed this tumour is probably one of the human solid tumour for which genetic alterations are found with highest prevalence. The mean number of chromosomal arm lost in pancreatic tumour cells is 14 out of the 39 autosomal segments. In more than $60 \%$ of pancreatic cancer one of the following chromosome arms are lost $1 p, 9 p, 17 p$ and 18q. Allelic losses on $3 p$, $6 p, 6 q, 8 p, 10 q, 12 q, 13 q, 18 p, 21 q$ and $22 q$ are observed in 30 to $60 \%$ of the cases. Associated with these losses, gains of chromosome arms are found in 16p, 20p, 17p and recurrent amplified regions are found in $6 \mathrm{q} 24,7 \mathrm{q} 22,12 \mathrm{p} 13^{[2]}$.

Allelic losses take part in the inactivation of tumor suppressors genes. In the most frequent chromosomal arm lost, the second copy of the tumor suppressor gene was found inactivated in $75 \%$ of the cases. In the $9 p$ the allelic loss target is p16 gene, which codes a protein that inhibits the complex Cyclin D-CdK4 and Cyclin D-cdk6 key players of cell cycle. In pancreatic adenocarcinoma p16 is inactivated in more than $90 \%$ of the cases. This bi-allelic inactivation is owing to homozygous deletion or by the association of allelic losses and inactivating point mutation or by hypermethylation of the enhancer region of the gene. In 17p the gene targeted by the deletion is TP53 and the prevalence of point mutation of this gene is up to $70 \%$ in adenocarcinoma of the pancreas. In chromosome $18 \mathrm{q}$ arm the characterization of homozygous deletion had allowed the identification of a tumour suppressor gene initially named DPC4 for deleted in pancreatic cancer. This gene had been renamed MADH4 (Mother Against Decapentaplegic Homolog) it codes for a protein that participates to the transduction of TGFß signaling pathway. This gene is inactivated in $35 \%$ of the cases by homozygous deletions or by the association of allelic loss and inactivating mutation in $20 \%$ of the cases ${ }^{[3]}$.

The tumor suppressor gene targeted by allelic loss occurring in the $1 p$ has not been yet identified; a most recent hypothesis suggests that the gene targeted could be a microRNA. 


\section{EGFR signalling pathways}

Others alterations lead to the activation of oncogenes. Among them, KRAS mutations in pancreatic cancer occur in more than $70 \%$ of the cases (Cosmic database, http://www.sanger.ac.uk/genetics/CGP/cosmic). These mutations occur in the first two nucleotides of codon 12, more rarely in codon 13 or 61 resulting in the constitutive activation of the MAPK and PI3K/AKT signal transduction pathways. Among other oncogenes implicated in pancreatic cancer a special mention must be made for the EGF receptor family. Oncogene HER2, overexpression in pancreatic cancer, has been reported to vary widely from 10 to $82 \%^{[4-6]}$, as HER1 is commonly expressed in pancreatic cancer.

This activation of EGF pathway leads to a cascade of phosphorylation in the Ras, $\mathrm{PI}(3) \mathrm{K}$ (phosphatidylinositol-3-OH kinase) and mTOR (mammalian target of rapamycin) signalling pathways which forms an intersecting biochemical network that, when mutated, drives cell growth in a manner unrestricted by environmental cues. Ultimately, these pathways drive tumorigenesis through the coordinated phosphorylation of proteins that directly regulate protein synthesis, cell-cycle progression and metabolism, and of transcription factors that regulate the expression of genes involved in these processes affecting cellular proliferation, angiogenesis, apoptosis, and metastatic spread ${ }^{[7]}$. Therefore the mTOR protein kinase has emerged as a critical growth control node, receiving stimulatory signals from Ras and $\mathrm{PI}(3) \mathrm{K}$ downstream from growth factors, as well as nutrient inputs in the form of amino-acid, glucose and oxygen availability. Notably, components of the Ras and $\mathrm{PI}(3) \mathrm{K}$ signalling pathways are mutated in most human cancers. The preponderance of mutations in these interconnected pathways suggests that the loss of growthcontrol checkpoints and promotion of cell survival in nutrient limited conditions may be an obligate event in tumorigenesis. Furthermore, since most of the recently clinically approved or developing targeted anticancer agents focused on partners of these signalling pathways, understanding the full circuitry of Ras, $\mathrm{PI}(3) \mathrm{K}$ and mTOR signalling pathways, including feedback loops, is a requirement for an understanding of the biological consequences of perturbing that pathway by targeted anticancer agents as inhibitors of mTOR or other $\mathrm{PI}(3) \mathrm{K}$ signalling components. Defining the combinations of inhibitors that are likely to be effective on tumors with specific combinations of genetic lesions will be an even greater challenge. However, on the basis of our increasing understanding of these networks, there is great hope that this strategy will ultimately be highly effective. As more drugs that target specific components of signal transduction pathways become available and as we increase our knowledge of the complexity of these signalling networks, the burden of selecting the correct drug combinations for each individual cancer patient will ultimately shift to the pathologist, who must identify the underlying defect in each tumor. This will require new diagnostic technologies and will be a major challenge over the next decade.

\section{KRAS mutations}

From the description of the repository of genetic alterations occurring in pancreatic cancer the alteration of EGFR pathway appears central either by the expression of the receptor itself or by the occurrence of KRAS mutation. Two classes of epidermal growth factor receptor (EGFR) inhibitors (antibodies ie antiEGFR or small molecules ie EGFR-TKI) have been tested in human cancers and from the 
experience from 2 others tumor locations we could have predicted the absence or the weak effectiveness of these treatments in pancreatic cancer patients. Indeed, in colorectal cancer, it has been now clearly shown that KRAS mutations were associated with resistance to antiEGFR (cetuximab and panitumumab) in patients with metastatic disease and that KRAS wild-type state predicts the efficacy of these treatments in terms of morphological objective response and survival ${ }^{[8-11]}$. In non small cell lung cancer KRAS mutation is an important predictor of poor response and outcome to EGFR-TKIs (erlotinib and gefitinib) ${ }^{[12]}$. In pancreatic cancer the addition of cetuximab to gemcitabine has not shown any benefit ${ }^{[13]}$ whereas the addition of erlotinib, another EGFR inhibitor, to gemcitabine has given positive results with a statistically significant improvement in overall survival ${ }^{14]}$. This drug has obtained approval in the USA and Europe. However the median survival increased in the experimental arm only of 10 days and difference between survival rate at 1 year increased from $17 \%$ to $23 \%$ leading to suggest a true benefit for only $6 \%$ of the patient. This study raises the issue of the status of KRAS for the patients that benefit from the treatment by erlotinib. The cost benefit of such treatment is largely dependant to the answer of this question.

\section{HER 2 inhibitors}

Concerning the approach of inhibiting HER2, the results are controversial and once again the absence of a well defined tumour population has probably impaired the results of the only phase II using trastuzumab a recombinant humanized IgG1 monoclonal antibody against HER2 in pancreatic cancer. There is evidence for positive in vitro and in vivo results in pancreatic cancer cell lines and in xenograft tumour models in a subgroup of tumour with high level HER2 protein $(3+)$ treated by trastuzumab ${ }^{[15]}$. However the only phase II using a combination of gemcitabine plus trastuzumab is negative showing only $6 \%$ response (2 of 32 cases) which is not superior to therapy with gemcitabine alone ${ }^{[16]}$. One of the explanations of this negative result could be that $90 \%$ (30 of 34 patients) of enrolled patients in the trial showed only a moderate (2+) expression of HER2, which is not the highest grade of expression. The true clinical advantage of trastuzumab in patients with high expression $(3+)$ of HER2 therefore remains unclear.

\section{MEK-1 and mTOR inhibitors}

New drugs targeting several proteins downstream from EGF receptor are in various stages of clinical development. In particular a selective small-molecule inhibitor (Cl-1040) of the dual-specificity kinases MEK (mitogen-activated protein kinase kinase) -1 and MEK-2 that is orally active has shown both target suppression and antitumour activity in a phase I study ${ }^{[17]}$. One partial response out of six patients with pancreatic cancer enrolled in this trial, has been observed. Furthermore the dual targeting of the EGFR and MAPK pathway by tarceva and Cl-1040 is associated with antitumor effects in four different pancreatic cancer xenografts with up-regulation and activation of both pathways that are resistant to each intervention separately, suggesting a synergistic effects of this combined therapeutic modality ${ }^{[18]}$. These results provide the basis to test EGFR inhibitors in combination with inhibitors of the MAPK in phase I and phase II trials for pancreatic cancer patients.

Furthermore as the Ras-MAPK and $\mathrm{PI}(3) \mathrm{K}$ pathways are strongly interconnected, 
disruption of one will, in many cases, push tumour cells to increase flux through the other in a virtual tug of war between proliferation and survival signals. One potential strategy to overcome this problem is to combine drugs against several relevant targets. For example combined inhibition of EGFR and $\mathrm{PI}(3) \mathrm{K}$ signalling in phosphatase and tensin homologue (PTEN)-mutated tumours has been shown to be a rational and therapeutically advantageous approach ${ }^{[19]}$. In this context there is some evidence that inhibitor of mTor (Mammalian Target of rapamycin) could be efficient in pancreatic cancer treatment. It has been shown that the signalling pathways regulating $\mathrm{mTOR}$ are frequently activated in pancreatic cancer, and that a rapamycin derivative (CCl-779; temsirolimus) could inhibit tumour growth in xenograft models as a single agent as well as combined with gemcitabine ${ }^{[20]}$. A least 2 ongoing trials are currently testing this agent in locally advanced or metastatic pancreatic cancer patients alone or in combination with gemcitabine (NCT00075647; NCT00593008).

\section{NF-kB inhibitors}

The PI3K mTor activation signalling pathways lead at the end to the activation of the ubiquitous transcription factor, nuclear factor kappa B (NF-kappaB) (for schematic representation see http://www.genome.ad.jp/kegg/pathway/hsa/hsa05212.html). NF-kB activation is important to pancreatic cancer growth and metastases. A large number of compounds are currently known as NF-kappaB modulators and include the isoprenoids, most notably kaurene diterpenoids and members of the sesquiterpene lactones class, several phenolics including curcumin and flavonoids such as silybin [21]. It has been shown in human pancreatic cancer cell lines that curcumin (diferuloylmethane) can suppress NF-KB, COX-2, PGE-2, IL-8 expression and cell proliferation in pancreatic malignancies, and all of these curcumin-induced changes are associated with potent proapoptotic effects suggesting a potential anti-tumoral effect in pancreatic cancer patients ${ }^{[22]}$. Furthermore Curcumin increased the inhibitory effect of gemcitabine on cell viability as well as its pro-apoptotic effect in COX-2 positive pancreatic cancer cell line ${ }^{[23]}$. After the proof of concept, three clinical trials testing curcumin (NCT00192842; NCT00094445; NCT00486460) in association with gemcitabine or celebrex are currently ongoing.

\section{Antisense Therapy}

Another therapeutic approach to target downstream effectors of the EGFR pathways is the antisense therapy. Antisense oligonucleotides are designed as a complimentary sequence of base pairs to a specific mRNA that in turn permits the sequence to bind to the mRNA and therefore block its expression. Different antisense oligonucleotides have been used targeting KRAS or HRAS in pancreatic cancer cell lines with promising results. ISIS-2503 is a phosphorothioate oligonucleotide antisense inhibitor of human $\mathrm{H}$-ras mRNA expression which has shown significant antitumor activity against a variety of human tumors implanted in athymic nude mice, including two pancreatic carcinoma cell lines. Furthermore in a phase II study the combination of gemcitabine and ISIS-2503 demonstrated activity in patients with pancreatic adenocarcinoma. In a patient population in which $90 \%$ of the patients had metastatic disease, a median overall survival of 6.7 months and an objective response rate of $10 \%$, including one complete response were observed suggesting a 
potential positive effect of this drug ${ }^{[24]}$.

\section{Conclusion}

Considerable excitement has arisen over the past years with the development and approval of a number of new therapeutic agents that have increased options and significantly improved outcomes of patients with cancer. However, recent randomized trials in pancreatic cancer gave poor results. It is becoming increasingly apparent that the complexity of the EGFR signalling cascade provides a wealth of mechanisms for resistance to EGFR targeted agents in patients with pancreatic cancer. Combination therapy with targeted agents should be efficient in these patients. Moreover, in order to select the best combination of targeted therapies we need to develop tools for the physician in order to identify the best treatment for a given patient and allowing the treatment "à la carte". The extensive characterization of genetic alterations of signalling pathways appeared to be a promising way in order to identify predictive factors of response to targeted therapies. 


\section{References}

**1. Croce CM. Oncogenes and cancer. N Engl J Med 2008; 358:502-11

Cancer is a DNA disease, and this recent paper gives a state of the art of the different genetic alterations of oncogenes tumor suppressor gene and microRNA found in tumor cells and underlines the clinical importance of them in the development of rational cancer treatment.

2. Hilgers W, Kern SE. Molecular genetic basis of pancreatic adenocarcinoma. Genes Chromosomes Cancer 1999; 26:1-12

*3. Maitra A, Kern SE, Hruban RH. Molecular pathogenesis of pancreatic cancer. Best Pract Res Clin Gastroenterol 2006; 20:211-26

This review depicted the prevalence of mutations occurring in various oncogenes and tumour suppressor gene in pancreatic cancer.

4. Novotny J, Petruzelka L, Vedralova J et al. Prognostic significance of c-erbB-2 gene expression in pancreatic cancer patients. Neoplasma 2001; 48:188-91

5. Safran H, Steinhoff M, Mangray $S$ et al. Overexpression of the HER-2/neu oncogene in pancreatic adenocarcinoma. Am J Clin Oncol 2001; 24:496-9

6. Stoecklein $\mathrm{NH}$, Luebke AM, Erbersdobler $\mathrm{A}$ et al. Copy number of chromosome 17 but not HER2 amplification predicts clinical outcome of patients with pancreatic ductal adenocarcinoma. J Clin Oncol 2004; 22:4737-45

**7. Shaw RJ, Cantley LC. Ras, $\mathrm{PI}(3) \mathrm{K}$ and mTOR signalling controls tumour cell growth. Nature 2006; 441:424-30

This review described an important network of interconnected signalling pathways Ras, $\mathrm{PI}(3) \mathrm{K}$ and mTOR which is activated by mutation in most human cancers suggesting that the loss of growth-control checkpoints and promotion of cell survival in nutrient-limited conditions may be an obligate event in tumorigenesis.

8. De Roock W, Piessevaux H, De Schutter $J$ et al. KRAS wild-type state predicts survival and is associated to early radiological response in metastatic colorectal cancer treated with cetuximab. Ann Oncol 2007; Epub ahead of print

9. Di Fiore F, Blanchard F, Charbonnier F et al. Clinical relevance of KRAS mutation detection in metastatic colorectal cancer treated by Cetuximab plus chemotherapy. Br J Cancer 2007; 96:1166-9

${ }^{* *} 10$. Lievre $A$, Bachet JB, Boige $V$ et al. KRAS mutations as an independent prognostic factor in patients with advanced colorectal cancer treated with cetuximab. J Clin Oncol 2008; 26:374-9

This series of colorectal cancer patients described the prognostic role of the somatic mutation of KRAS in colorectal metastatic cancer patients treated by cetuximab. This is the largest series published up to now, showing the clinical importance of the determination of KRAS status in colorectal cancer patients before treatment with an antiEGFR targeted therapy

11. Lievre A, Bachet JB, Le Corre $D$ et al. KRAS mutation status is predictive of response to cetuximab therapy in colorectal cancer. Cancer Res 2006; 66:3992-5

*12. Massarelli E, Varella-Garcia M, Tang X et al. KRAS mutation is an important predictor of resistance to therapy with epidermal growth factor receptor tyrosine kinase inhibitors in non-small-cell lung cancer. Clin Cancer Res 2007; 13:2890-6

This series of non-small-cell lung cancer shows that KRAS is an important predictor of resistance to tarceva a small molecule inhibitor of the tyrosine kinase domain of the EGF receptor 
13. Cascinu S, Berardi R, Labianca R et al. Cetuximab plus gemcitabine and cisplatin compared with gemcitabine and cisplatin alone in patients with advanced pancreatic cancer: a randomised, multicentre, phase II trial. Lancet Oncol 2008; 9:3944

${ }^{* *}$ 14. Moore MJ, Goldstein D, Hamm J et al. Erlotinib plus gemcitabine compared with gemcitabine alone in patients with advanced pancreatic cancer: a phase III trial of the National Cancer Institute of Canada Clinical Trials Group. J Clin Oncol 2007; 25:1960-6

This paper show for the first time in a phase III trail the benefit of adding a targeted therapy to gemcitabine for the treatment. The tested arm benefit is real but modest.

*15. Kimura K, Sawada T, Komatsu M et al. Antitumor effect of trastuzumab for pancreatic cancer with high HER-2 expression and enhancement of effect by combined therapy with gemcitabine. Clin Cancer Res 2006; 12:4925-32

The antitumor effect of trastuzumab in pancreatic cancer with high HER-2 expression was shown in vitro and in vivo in this paper leading to suggest that in the subset of pancreatic cancer with 3+ HER-2 expression, trastuzumab could be of clinical importance.

16. Safran $H$, lannitti $D$, Ramanathan $R$ et al. Herceptin and gemcitabine for metastatic pancreatic cancers that overexpress HER-2/neu. Cancer Invest 2004; 22:706-12

17. Lorusso PM, Adjei AA, Varterasian $\mathrm{M}$ et al. Phase I and pharmacodynamic study of the oral MEK inhibitor $\mathrm{Cl}-1040$ in patients with advanced malignancies. J Clin Oncol 2005; 23:5281-93

*18. Jimeno A, Rubio-Viqueira B, Amador ML et al. Dual mitogen-activated protein kinase and epidermal growth factor receptor inhibition in biliary and pancreatic cancer. Mol Cancer Ther 2007; 6:1079-88

This paper underlines the importance of studying the signalling pathway in its whole in order to build efficent strategy by combining different targeted therapy. They demonstrated that treatment associating an anti EGFR with a MAPK inhibitor was more effective than either single agent alone in pancreatic xenograft tumour model.

19. She QB, Solit DB, Ye Q et al. The BAD protein integrates survival signaling by EGFR/MAPK and PI3K/Akt kinase pathways in PTEN-deficient tumor cells. Cancer Cell 2005; 8:287-97

20. Ito $\mathrm{D}$, Fujimoto $\mathrm{K}$, Mori $\mathrm{T}$ et al. In vivo antitumor effect of the mTOR inhibitor $\mathrm{CCl}-779$ and gemcitabine in xenograft models of human pancreatic cancer. Int J Cancer 2006; 118:2337-43

21. Bremner $P$, Heinrich $M$. Natural products as targeted modulators of the nuclear factor-kappaB pathway. J Pharm Pharmacol 2002; 54:453-72

22. Li L, Aggarwal BB, Shishodia $S$ et al. Nuclear factor-kappaB and IkappaB kinase are constitutively active in human pancreatic cells, and their down-regulation by curcumin (diferuloylmethane) is associated with the suppression of proliferation and the induction of apoptosis. Cancer 2004; 101:2351-62

23. Lev-Ari S, Vexler A, Starr A et al. Curcumin augments gemcitabine cytotoxic effect on pancreatic adenocarcinoma cell lines. Cancer Invest 2007; 25:411-8

24. Alberts SR, Schroeder M, Erlichman C et al. Gemcitabine and ISIS-2503 for patients with locally advanced or metastatic pancreatic adenocarcinoma: a North Central Cancer Treatment Group phase II trial. J Clin Oncol 2004; 22:4944-50 